\title{
Integración de las matemáticas y las ciencias bibliotecológica y de la información: una necesidad en los nuevos planes de estudio de la licenciatura
}

\author{
ROSA MA RÍA MARTÍNEZ RIDER \\ SALVADORGORBEA-PORTAL \\ EscueladeBibliotecologíaeInformación,UniversidadAutónoma \\ de San Luis Po to síC.P.78494, San Luis Po to sí, México. \\ Tel. (52) 48 18- 2521, Fax: (52) 48 18- 2475 \\ E-mail:licbib@ biblos.dgb.uaslp.mx \\ Centro Universitario deInvestigacionesBibliotecológicasdela UNAM, \\ To rre II deHu mani dades Piso 12, CU, C.P. 04510, México, D .F., \\ Tel. (525) 623- 0360 Fax: (525) 550- 7461 \\ E-mail:portal@ servidor.unam.mx
}

Trabajo recibido

el 15 de agosto

de 1997

Trabajo aceptado

el 8 de enero de 1998

\section{RESUMEN}

Seexponenlosan teceden tes deluso delosméto doscuantitativosenlascienciasso cialesy, como una con secuen ciadeéste, el pro ceso dein tegración que se manifiesta entre las matemáticas y las ciencias bibliotecológica y de la información, con elin terés dedestacarlaim portan ciaquetienelavin culación delosméto dosymo delosmatemáticosyestadísticosenlosplanesdeestudio de la licenciatura en este campo. Se presenta como estudio de caso de este análisislareestructuración del plan curricular de la Licenciatura en Bibliotecología e Información de la Universidad Autónoma de San Luis Potosí en México.

\section{ABST RACT}

Thehistoryof theuse of quan titativemethodsin theso cial scien cesispresented and, as a con sequen ce of this, the pro cess of in te gration bet ween mathe maticsandlibraryand informationscien ces, in orderto stress theim portan ce of the links between mathematical and statistical methods in the undergraduate syllabus in this field of study. The restructuring of the undergraduate coursein Libraryand In formation Scien ceat theA uto no mous Universityof San Luis Potosi in Mexico is used as a case study for this type of analysis. 


\section{INTRODUCCIÓN}

Tno de los antecedentes más evidentes en la introducción de las matemáticas a las disciplinas sociales, con el propósito de darle legitimidad de ciencia, se encuentra en la doctrina de Augusto Comte, quien hizo importantes aportaciones en sus planteamientos a la investigación científica y propició con ello la sistematización y fomento de la especialización de las ciencias.

Comte denominó a su doctrina epistemológica "positivismo," sin embargo, las obras filosóficas destacan que este término debe usarse con cautela para no confundirlo con otras corrientes de pensamiento y evitar ambigüedades. Esta doctrina, que se caracteriza por rechazar contundentemente las nociones a prioi y los conceptos universales, admite como única fuente del conocimiento a la experiencia sensible, ésta debe provenir del campo de lo positivo, esto es, de lo que es observable, medible y experimentable, por lo tanto, no acepta el conocimiento obtenido desde la metafísica o la teología por no incluir, bajo ninguna circunstancia, a la especulación.

Esta doctrina ha tenido una fuerte influencia en los siglos XIX y XX y aunque ha hecho grandes aportaciones a la ciencia, también se le ha cuestionado severamente en este siglo.

El sociólogo Emile Durkheim quien "[...]es ampliamente conocido por su insistencia en distinguir, por un lado, las predicciones de sentido común y la ideología, y por el otro, el conocimiento rigurosamente científico"(Bravo, 1980, p.15) concluyó que se deben investigar los hechos sociales como "cosas," es decir, por sus características objetivas, así D urkheim trató de darle cientificidad a las disciplinas sociales, pero a la par, neutralizar los aspectos axiológicos y éticos del individuo. Durkeim, sin embargo, le da un nuevo enfoque a los principios comtianos, pues permite que se diferencien las disciplinas unas de otras.

La incursión de las matemáticas en lo social, a partir de un enfoque cuantitativo desde el paradigma empíreo-analítico, fue una novedad, pues genera una explicación a la causalidad de los fenómenos sociales, y define así una posición científica. Aunque en ocasiones se opine lo contrario, las matemáticas se encuentran presentes en todo tipo de conocimiento: científico o empírico-espontáneo y cotidiano.

El paradigma empíreo-analítico se caracteriza por el uso del método científico y es el resultado de los planteamientos positivistas, pero enriquecidos y cuestionados, específicamente utiliza en sus investigaciones el método hipotético-deductivo o el inductivo, puesto que su uso le da reconocimiento y prestigio a un trabajo de investigación, cuyo proceso pretende comprobar una hipótesis y se orienta al manejo de variables para llegar a ciertos resultados que comprueben 0 no los supuestos.

Los hechos sociales pretenden comprobarse en la realidad y así elaborar teorías y leyes. Aluden a la "neutralidad" y tratan de pronosticar fenómenos. 
Un rasgo muy peculiar de este paradigma se refiere a su carácter cognitivo-analítico en la obtención de conocimiento. A unque en este paradigma "[...]]lo concreto pierde su riqueza de contradictoriedad y multiplicidad de significados" (Bravo, 1980, p.22); lo cierto es que se trata de una forma de conocimiento que permite la explicación de los hechos o fenómenos sociales, además de acentuar el carácter científico de las disciplinas sociales. Permite también desarrollar importantes habilidades de razonamiento y construir conocimiento a partir de los datos, rescatando junto con los aspectos cuantitativos a los cualitativos para un mejor entendimiento de los problemas sociales.

Los hechos y fenómenos sociales implicados en las actividades bibliotecaria y de información, como por ejemplo la generación y uso de la información, no escapan de este enfoque, ya generalizado en las ciencias sociales; la incursión de los métodos cuantitativos (como complemento de los cualitativos), desde el paradigma empíreo-analítico, ya ha aportado resultados enriquecedores al cuerpo teórico de las disciplinas científicas que estudian y sustentan su comportamiento, esta perspectiva se presenta en la actualidad no sólo como una atractiva línea de investigación en esta esfera, sino como una exigencia en la formación y desempeño de sus profesionales.

\section{LAS MATEMÁTICAS Y LAS CIEN CIAS BIBLIOTECOLÓGICA Y DE LA INFORMACIÓN}

La integración del conocimiento matemático a las ciencias bibliotecológica y de la información ha propiciado, entre otros aspectos, la confrontación entre el conocimiento teórico de estas disciplinas y la realidad empírica en la que se desarrollan las actividades bibliotecarias y de información, es decir, esta integración ha propiciado, en un sentido, el fortalecimiento de postulados teóricos y, en el otro, ha orientado el desempeño de la práctica.

La modelación matemática de un objeto o fenómeno de la realidad en estas actividades, como es el caso del comportamiento de la circulación de documentos en una biblioteca, permite identificar determinadas regularidades internas de este fenómeno que ayudan a pronosticar su comportamiento futuro y por consiguiente comprobar supuestos sobre la construcción de principios teóricos generales que enriquezcan las propias disciplinas científicas.

La incursión de los métodos cuantitativos a estas actividades, como método científicamente comprobado, desde el paradigma empíreo-analítico ha contribuido en alguna medida, junto con otros aspectos como los tecnológicos, a reducir las incesantes polémicas sobre la identidad científica de las disciplinas bibliotecológica y de la información.

Budd (1995) señala que en bibliotecología, posiblemente más por accidente que por intención, la epistemología positivista es la que ha regido el trabajo de in- 
vestigación; una prueba de ello se puede observar en el carácter empíreo-analítico que ha primado en la mayoría de las investigaciones realizadas en este campo y la proliferación de métodos cuantitativos, desde principios de siglo, en el análisis y comprobación del comportamiento de regularidades y en la búsqueda de soluciones a problemas surgidos en la práctica bibliotecaria y de información.

Lo anterior se manifiesta en la consideración de que las matemáticas son un elemento importante dentro de las ciencias bibliotecológica y de la información porque como se mencionó, al igual que en el resto de las ciencias sociales, proporcionan una forma de conocimiento a través de la lógica y la razón que le permite al profesional de esta esfera representar la realidad objetiva y profundizar en su conocimiento y sus relaciones. Como bien se ha señalado "las matemáticas además de aplicarse para la medición en la investigación científica se emplean también para establecer la existencia de relaciones" (Gómez y Peralta 1995, p.37).

Las matemáticas abren un campo amplio de posibilidades para las ciencias bibliotecológica y de la información, puesto que aportan resultados dirigidos a la toma de decisiones en materia de política científica y de información, lo que facilita la planeación de su dinámica social, proporcionan criterios de eficiencia, eficacia (técnica y económica) y de calidad, así como la denominada mejora continua.

Un factor determinante en todo este escenario lo constituye el bibliotecólogo o profesional de la información, quien desde diferentes esferas profesionales -la docencia, la investigación o los servicios- contribuye a este proceso de matematización. Es a él a quien le corresponde esta tarea de integración y no a los matemáticos, como se piensa y sucede en ocasiones, porque éste es un proceso y una exigencia que se manifiesta cada vez con más énfasis, en las actividades bibliotecaria y de la información y no surge como una necesidad de las matemáticas (Gorbea, 1995).

Es de suponer, como sucede en todo nivel de integración disciplinar, que el bibliotecólogo o profesional de la información tendrá que apoyarse y relacionarse en forma interdisciplinaria con matemáticos y estadísticos para enfrentar juntos esta tarea compleja, que se manifiesta, al igual que la tecnología de cómputo, como una exigencia del mercado de trabajo y un nuevo reto para la formación integral del profesional de esta esfera.

Para poder enfrentar este desafío será menester comenzar con la incorporación o fortalecimiento de las matemáticas en los planes y programas de estudio en el ámbito de las licenciaturas, planteadas éstas como uno de los objetivos instructivos que hay que cumplimentar en la formación del profesional, y continuar su fortalecimiento en la formación posgraduada de maestría y doctorado.

A continuación, como caso de estudio de las anteriores reflexiones, se presenta la incorporación de las matemáticas y los estudios métricos de la información al 
plan de estudio de la licenciatura en bibliotecología e información de la Universidad Autónoma de San Luis Potosí en México.

\section{LAS MATEMÁTICAS EN EL CURRÍCULUM DE LA ESCUELA DE BIBLIOTECOLOGÍA E INFORMACIÓN DE LA UNIVERSIDAD AUTÓNOMA DE SAN LUIS POTOSÍ}

El 18 de marzo de 1998 fue aprobado el "Proyecto de Reestructuración Curricular," de la Escuela de Bibliotecología e Información de la Universidad Autónoma de San Luis Potosí, con este proyeto se culminó el esfuerzo de varios años de trabajo por parte de una comisión curricular coordinada y asesorada por los autores de este artículo respectivamente e integrada por un grupo de experimentados profesores de esta escuela. (Una información más completa sobre la reestructuración curricular puede ser consultada en: Proyeto de restructuración amiaular..., 1998.)

Esta escuela tiene la misión de formar profesionales con calidad para seleccionar, organizar, sistematizar, conservar y difundir la información documental, mediante distintas prácticas sociales, desarrollando la capacidad de provocar, generar y adaptarse al cambio que de la bibliotecología demanda la sociedad a través de una constante innovación, actualización y espíritu de superación.

El proyecto de Licenciado en Bibliotecología pretende formar un profesional de calidad que inmerso en un modelo neoliberal sea capaz de elaborar su propia propuesta ante la problemática que prevalece en el país y ante la ausencia de utopía.

D espués de tres años de trabajo curricular en el que se revisó y evaluó la congruencia interna (objetivos curriculares, plan de estudios, perfil de ingreso, etcétera) y la externa (seguimiento de egresados, encuesta a empleadores y mercado de trabajo y práctica profesional), además de reuniones con expertos en el tema de consultar los planes y programas de las escuelas de bibliotecología del país, de una autoevaluación diagnóstica que abarcó la problemática, oportunidades, amenazas, retos y perspectivas de la actual escuela de bibliotecología e información, se determinó la organización por ejes curriculares que, por un lado, permiten desarrollar un pensamiento crítico y, por el otro, desarrollar conocimientos, habilidades y actitudes transversas, articuladas con la realidad social en el momento histórico que se vive.

Los cuatro ejes curriculares son:

\section{Eje social}

Abarca el conocimiento del contexto en sus variadas dimensiones (histórico, político, económico, cultural, etcétera), así como el papel que desempeñan las unidades de información (bibliotecas, archivos, centros de información o docu- 
mentación) dentro de la sociedad. Incluye lo referente a los procesos personales, grupales, colectivos y electrónicos de comunicación.

Se fomentará el espíritu de servicio, la solidaridad, las relaciones humanas positivas, la cooperación, el trabajo en equipo, la participación colectiva de las decisiones, el análisis de las políticas nacionales de información, sus repercusiones en la industria y mercado de la información y las nuevas tecnologías en los servicios, entre otras cosas.

\section{Eje humanístico}

Incluye los elementos que permiten al estudiante desarrollar una actitud creativa, crítica y comprometida, mediante el análisis de los actuales valores culturales, rescatando los planteamientos básicos de la axiología y la ética, así como el conocimiento crítico en relación con el desarrollo del país.

Se fomentarán actitudes de iniciativa, decisión, apertura, tolerancia, autodesarrollo y superación. Se analizarán los elementos que propician la aculturación y los valores como la justicia, el derecho a la información, respeto a nuestra patria.

\section{Eje metodológico}

Pretende proporcionar los elementos para la aplicación, interpretación y comprensión de los fenómenos bibliotecológicos en diferentes niveles y dimensiones; asimismo brindar las herramientas para la presentación de propuestas, programas y proyectos de investigación; y fomentar el uso de las metodologías cualitativas y cuantitativas.

\section{Eje disciplinar}

Comprende cinco subejes:
A. Fundamentos
B. Análisis y organización de la información
C. Servicios de información
D. Economía y administración de la información
E. Tecnología de información

Éstos pretenden proporcionar elementos sobre el conocimiento y la función social de la disciplina, así como para el manejo de instrumentos que faciliten la comprensión de conocimientos, el desarrollo de habilidades y la elección de actitudes en el desempeño de su práctica profesional, que guían el trabajo en las diferentes unidades de información, proporcionando una visión holística de la disciplina en relación con el entorno y considerando la explosión de información, las nuevas tecnologías en las bibliotecas electrónicas y virtuales, la importancia de los procesos documentales en la difusión de variados servicios bibliotecarios en el ámbito general o específico y el requerimiento para construir conocimiento bibliotecológico. 
ESCUELA DE BIBLIOTECOLOGÍA E INFORMACIÓN

Licenciado en Bibliotecologíae Información

EJES

\begin{tabular}{|c|c|c|}
\hline SOCIAL & HUMANÍSTICO & METODOLÓGICO \\
\hline $\begin{array}{l}\text { INTRODUCCIÓN A LA } \\
\text { PSICOLOGÍA }\end{array}$ & $\begin{array}{l}\text { INTRODUCCIÓN A LA } \\
\text { FILOSOFİA }\end{array}$ & MATEMÁTICAS \\
\hline $\begin{array}{l}\text { INTRODUCCIÓN A LA } \\
\text { PEDAGOGÍA }\end{array}$ & $\begin{array}{l}\text { TALLER DE LECTURA } \\
\text { Y REDACCIÓN }\end{array}$ & $\begin{array}{l}\text { PROBABILIDADESY } \\
\text { ESTADÍSTICAS }\end{array}$ \\
\hline $\begin{array}{l}\text { TEORÍA DE LA } \\
\text { COMUNICACIÓN }\end{array}$ & $\begin{array}{l}\text { HISTORIA DE LA } \\
\text { CULTURA I y II }\end{array}$ & $\begin{array}{l}\text { METODOLOGÍA DE LA } \\
\text { INVESTIGACIÓN }\end{array}$ \\
\hline RELACIONES HUMANAS & $\begin{array}{l}\text { HISTORIA DE LA } \\
\text { CIENCIA }\end{array}$ & $\begin{array}{l}\text { SEMINARIO DE } \\
\text { INVESTIGACIÓN } \\
\text { BIBLIOTECOLÓGICA }\end{array}$ \\
\hline $\begin{array}{l}\text { ADMINISTRACIÓN } \\
\text { GENERAL }\end{array}$ & $\begin{array}{l}\text { HISTORIA DEL LIBRO } \\
\text { Y LAS BIBLIOTECAS }\end{array}$ & \\
\hline $\begin{array}{l}\text { ADMINIST RACIÓN DE } \\
\text { RECURSOS HUMANOS }\end{array}$ & $\begin{array}{l}\text { SEMINARIO DE } \\
\text { HISTORIA DE MÉXICO }\end{array}$ & \\
\hline $\begin{array}{l}\text { LEGISLACIÓN Y } \\
\text { NORMALIZACIÓN }\end{array}$ & & \\
\hline $\begin{array}{l}\text { CONTEXTO DE LA } \\
\text { INFORMACIÓN EN } \\
\text { MÉXICO I Y II }\end{array}$ & & \\
\hline $\begin{array}{l}\text { DISEÑO DE EDIFICIOS } \\
\text { PARA UNIDADES DE } \\
\text { INFORMACIÓN }\end{array}$ & & \\
\hline
\end{tabular}




\begin{tabular}{|c|c|c|c|c|}
\hline \multicolumn{5}{|c|}{ D I S C I P L I N A R } \\
\hline \multicolumn{5}{|c|}{ S U BE J E S } \\
\hline Fundamentos & $\begin{array}{l}\text { Análisis y orga- } \\
\text { nización de la } \\
\text { infomación }\end{array}$ & $\begin{array}{l}\text { Senvicios de in- } \\
\text { formación }\end{array}$ & \begin{tabular}{|c} 
Economía y ad- \\
ministracion \\
de la \\
información
\end{tabular} & $\begin{array}{c}\text { Tecnología de } \\
\text { información }\end{array}$ \\
\hline $\begin{array}{l}\text { Introducción a la } \\
\text { bibliotecología }\end{array}$ & $\begin{array}{l}\text { Catalogación } \\
\text { I, II, III, IV, V }\end{array}$ & $\begin{array}{l}\text { Fuentes de } \\
\text { información } \\
\text { I, II, III }\end{array}$ & $\begin{array}{l}\text { Administración } \\
\text { de unidades de } \\
\text { información }\end{array}$ & Computación \\
\hline Archivología & $\begin{array}{l}\text { Clasificación } \\
\text { I, II, III }\end{array}$ & $\begin{array}{l}\text { Usuarios de la } \\
\text { información }\end{array}$ & $\begin{array}{l}\text { Planificación } \\
\text { estratégica de } \\
\text { unidadesde } \\
\text { infomación }\end{array}$ & $\begin{array}{l}\text { Análisisde } \\
\text { sistemas }\end{array}$ \\
\hline $\begin{array}{l}\text { Teonía del } \\
\text { conocimiento } \\
\text { bibliológico } \\
\text { informativo }\end{array}$ & $\begin{array}{l}\text { Tallerde } \\
\text { conservación y } \\
\text { restauración }\end{array}$ & $\begin{array}{l}\text { Fondos de } \\
\text { información y } \\
\text { referencia }\end{array}$ & $\begin{array}{l}\text { Economía de la } \\
\text { información }\end{array}$ & $\begin{array}{l}\text { Automatización } \\
\text { de unidades de } \\
\text { información }\end{array}$ \\
\hline $\begin{array}{l}\text { Bibliotecología } \\
\text { comparada }\end{array}$ & $\begin{array}{l}\text { Indización y } \\
\text { lenguajesde } \\
\text { búsqueda } \\
\text { informativa }\end{array}$ & $\begin{array}{l}\text { Serviciosde } \\
\text { información } \\
\text { I, II, III }\end{array}$ & & $\begin{array}{l}\text { Redes y sistemas } \\
\text { de información }\end{array}$ \\
\hline & $\begin{array}{l}\text { Redacción de } \\
\text { documentos } \\
\text { científicos }\end{array}$ & & & $\begin{array}{l}\text { Nuevas } \\
\text { tecnologías de } \\
\text { información }\end{array}$ \\
\hline & $\begin{array}{l}\text { Taller de } \\
\text { procesamiento } \\
\text { de información }\end{array}$ & & & \\
\hline & $\begin{array}{l}\text { Bibliografía } \\
\text { I, II }\end{array}$ & & & \\
\hline & $\begin{array}{l}\text { Bibliografía } \\
\text { mexicana }\end{array}$ & & & \\
\hline
\end{tabular}




\begin{tabular}{|c|c|c|c|c|c|c|c|c|c|}
\hline \multirow{8}{*}{$\begin{array}{l}0 \\
0 \\
01 \\
01 \\
0 \\
0 \\
0 \\
0 \\
0 \\
0 \\
0 \\
0 \\
0 \\
0 \\
0 \\
0 \\
0\end{array}$} & 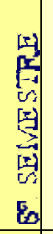 & 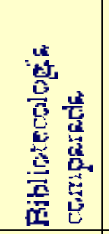 & 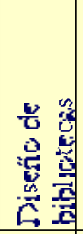 & 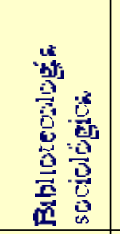 & 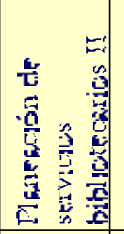 & 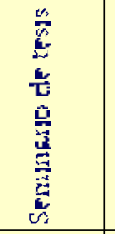 & & & \\
\hline & 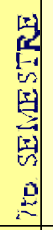 & 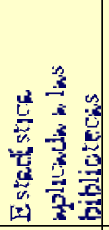 & 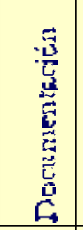 & 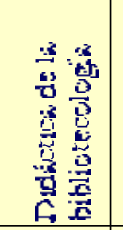 & 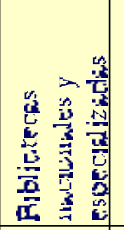 & 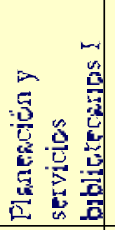 & 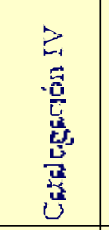 & 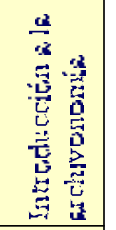 & \\
\hline & 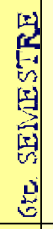 & 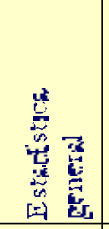 & 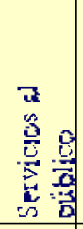 & 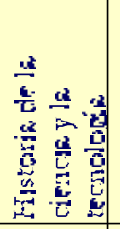 & 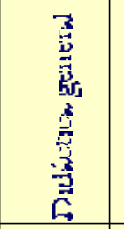 & 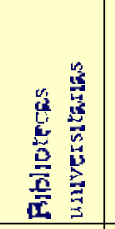 & 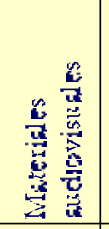 & 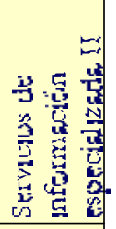 & \\
\hline & 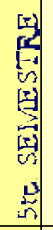 & 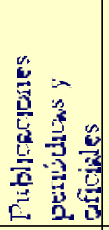 & 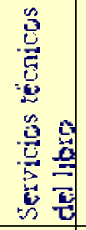 & 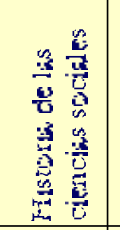 & 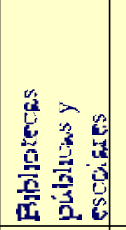 & 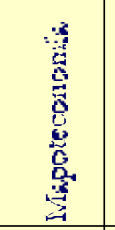 & 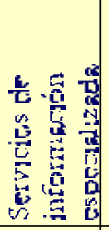 & 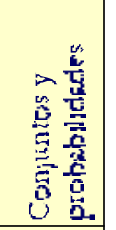 & \\
\hline & 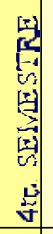 & 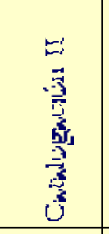 & 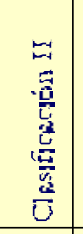 & 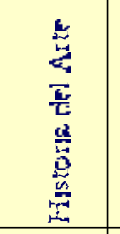 & 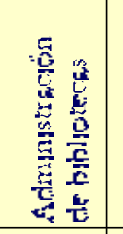 & 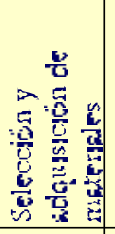 & 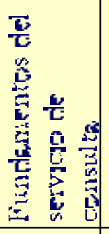 & 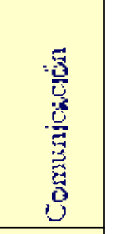 & \\
\hline & 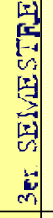 & 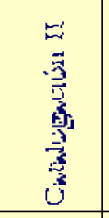 & 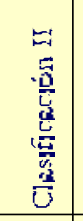 & 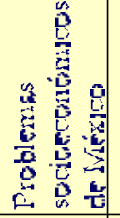 & 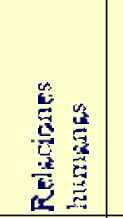 & 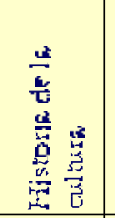 & 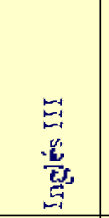 & 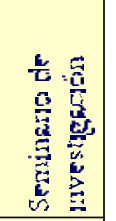 & \\
\hline & 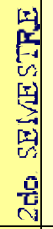 & 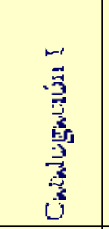 & 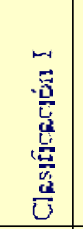 & 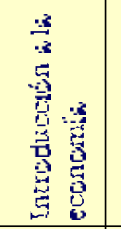 & 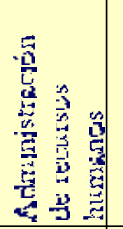 & 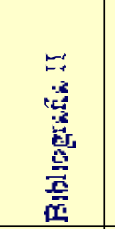 & 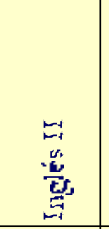 & 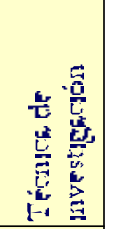 & \\
\hline & 亦 & 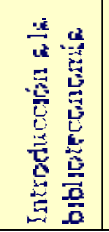 & 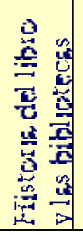 & 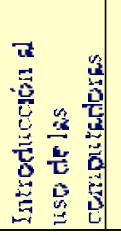 & 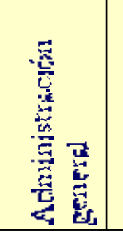 & 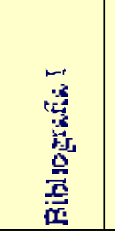 & 焉 & 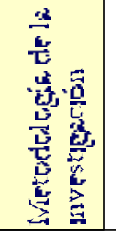 & \\
\hline
\end{tabular}


El plan de estudios anterior incluyó las materias de:

1.Conjuntos y probabilidades como apoyo a la introducción a la computación.

2. Estadística general y Estadística aplicada a las bibliotecas.

Sin embargo, después de la evaluación se concluyó lo siguiente:

1. Conjuntos y probabilidades se impartía en el 5o semestre e Introducción a la computación se cambió a $1^{\underline{0}}$ semestre, por tanto, se convirtió en una materia vacía.

2. Las estadísticas requerían de una materia previa de matemáticas.

3. Las estadísticas debían apoyar a las materias de Métodos de investigación, Técnicas de investigación; y al Seminario de investigaciones bibliotecológicas, mismas que se impartían en $1^{0}, 2^{\circ}$ y $3^{3}$ semestres, por tanto, los alumnos tenían una gran dificultad para aplicar técnicas de investigación de campo.

4. Las necesidades laborales exigen prácticas profesionales nuevas donde las matemáticas juegan un papel importante para la gestión, planeación y toma de decisiones.

5. La bibliotecología se construye mediante diversas formas de conocimiento, una de éstas se genera a través del paradigma empíreo-analítico, y esta investigación es prioritaria para acceder de distintas formas a la realidad, a través del método cuantitativo.

6. La formación integral abarca también los conocimientos, habilidades y actitudes que proporcionan las matemáticas para que se lleven a la práctica en situaciones y contextos diversos, así como en diferentes actividades (administrativas, de investigación, planificación, etcétera).

7. La matemática es una herramienta básica de aplicación.

8. Los métodos cuantitativos recuperan los aspectos cualitativos para darle un sentido y un significado al número con la finalidad de resolver problemas.

Dentro del eje metodológico antes mencionado, uno de los objetivos del proyecto académico es: aplicar las metodologías de investigación cualitativa y cuantitativa a problemas bibliotecológicos y de información teóricos o prácticos.

Entre las características de egreso se citan las siguientes que también se desarrollan por medio de las matemáticas: análisis, comparación, clasificación, identificación, creatividad, razonamiento, evaluación y aplicación de técnicas.

El nuevo plan de estudios (ver Mapa Curricular en p.128 y 129), después del análisis que realizó la Comisión Curricular, considera las siguientes materias en la formación matemática del licenciado en bibliotecología e información.

* Matemáticas

* Probabilidades y estadísticas

* Fuentes de información II (métrica)

* Usuarios de la información

* Servicios de información I, II, III (parte del programa)

* Diseño de edificios para unidades de información

* Economía de la información 
Metodología de la investigación (cuantitativa)

Las materias de usuarios de la información, servicios de información I, II y III, contienen una parte que corresponde a los aspectos cualitativos.

La materia de matemáticas tiene como objetivo proporcionar los elementos para aplicar los métodos y modelos matemáticos a distintas áreas de la bibliotecología. En el campo laboral es el fundamento para los estudios métricos de información; para el análisis documental; para identificar los flujos de información; para la gestión administrativa y para evaluar el desarrollo de las unidades de información, que apoyan la planificación y la toma de decisiones.

El enfoque teórico-práctico de la asignatura Probabilidades y Estadísticas permitirá incorporar al campo integral del estudiante, los elementos necesarios para comprender la importancia de la información estadística y probabilística a fin de que aplique conocimientos y habilidades en el diseño de programas y estrategias para mejorar y/ o desarrollar servicios y recursos informativos de acuerdo con las exigencias del campo profesional.

Fuentes de Información II contribuye a desarrollar la capacidad de racionamiento métrico en los estudiantes y los dota de herramientas, de fuentes y recursos de información. El bibliotecólogo, al igual que cualquier científico, requiere de información y datos mensurables para enfrentarse a la toma de decisiones en la gestión de diversos procesos y operaciones unitarias que se desarrollan cotidianamente en la actividad bibliotecaria, para ello, deberán contar con el conocimiento necesario sobre métodos y modelos bibliométricos e informétricos que le permitan la evaluación de las fuentes de información, así como el análisis de las regularidades de los flujos de información documental.

La materia de Metodología de investigación tiene la finalidad de aplicar los conocimientos en una investigación aplicada, asimismo de proporcionar los elementos para la presentación de proyectos, programas y propuestas.

También las matemáticas son un apoyo a una parte de las siguientes materias: Usuarios de la información; Servicios de información I, II y III; Diseño de edificios para unidades de información; Planificación estratégica de unidades de información; Economía de la información, y Análisis de sistemas, automatización de unidades de información, redes de información y nuevas tecnologías de información.

Otras materias como complemento de la investigación cuantitativa tienen la finalidad de proporcionar los elementos para la aplicación en investigaciones cualitativas a partir de métodos etnográficos, pues insistimos en que la bibliotecología debe construir su conocimiento en diferentes formas, que partan de varios paradigmas de investigación social. 


\section{Mapa Cumiailar}

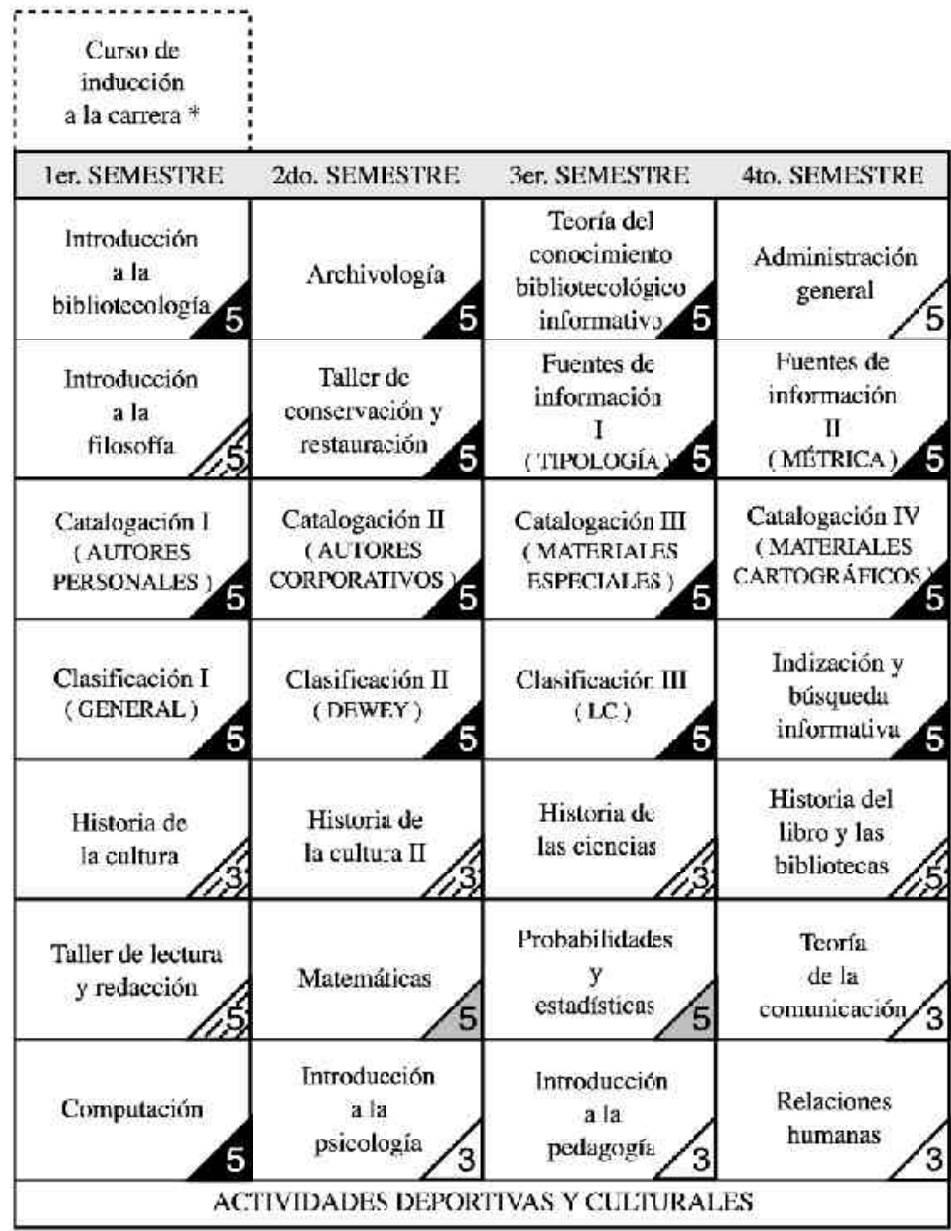

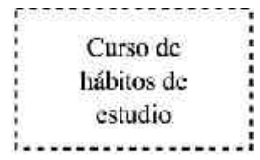

* CURSO EXTRACURRICULAR OBLIGATORIO
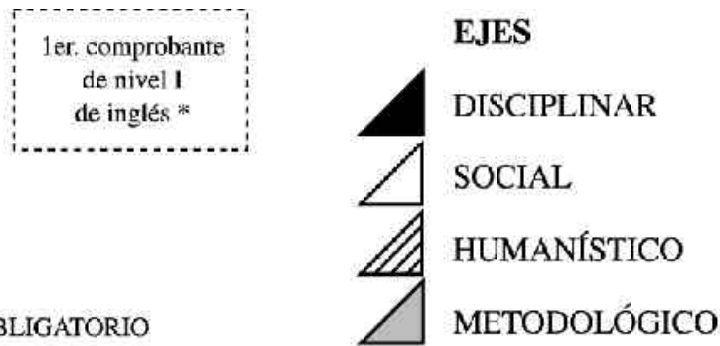


\section{Mapa Cumiaular}

\begin{tabular}{|c|c|c|c|c|}
\hline 5to. SEMESTRE & 6to. SEMESTRE & 7mo. SEMESTRE & 8vo. SEMESTRE & $\begin{array}{l}\text { YODALIDADES } \\
\text { DE TITULACIOS }\end{array}$ \\
\hline $\begin{array}{l}\text { Administración de } \\
\text { recursos } \\
\text { humanos }\end{array}$ & $\begin{array}{l}\text { Administración de } \\
\text { unidades de } \\
\text { información }\end{array}$ & $\begin{array}{l}\text { Planificación } \\
\text { estratégica de } \\
\text { unidades de } \\
\text { información }\end{array}$ & $\begin{array}{l}\text { Eccnomía de la } \\
\text { información }\end{array}$ & \multirow{7}{*}{ 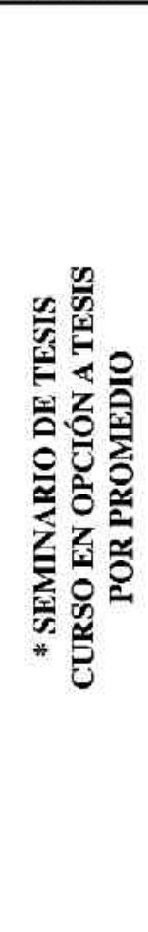 } \\
\hline $\begin{array}{l}\text { Fuentes de } \\
\text { información III } \\
\text { (SELECCIÓNY } \\
\text { ADQUISICIÓN) }\end{array}$ & $\begin{array}{l}\text { Servicios de } \\
\text { información I } \\
\text { (GENERALES) }\end{array}$ & $\begin{array}{l}\text { Servicios de } \\
\text { información II } \\
\text { (INFORMACIÓN } \\
\text { Y RFFFRENCIA }\end{array}$ & $\begin{array}{c}\text { Servicios de } \\
\text { información III } \\
\text { (EVALUACIÓN }\end{array}$ & \\
\hline $\begin{array}{l}\text { Catalugation V } \\
\text { (PUBLICACIONES } \\
\text { PERIÓDICAS) }\end{array}$ & $\begin{array}{c}\text { Taller de } \\
\text { procesumiento } \\
\text { información }\end{array}$ & $\begin{array}{l}\text { Discño de } \\
\text { edificios para } \\
\text { unidades de }\end{array}$ & $\begin{array}{c}\text { Bibliotecologia } \\
\text { comparada }\end{array}$ & \\
\hline $\begin{array}{l}\text { Redacción de } \\
\text { documentos } \\
\text { científicos }\end{array}$ & Bibliograffa I & Bibliografi: & $\begin{array}{l}\text { Bihlingrafía } \\
\text { mexicana }\end{array}$ & \\
\hline $\begin{array}{c}\text { Seminario de } \\
\text { historia de } \\
\text { México }\end{array}$ & $\begin{array}{l}\text { Legislación } \\
\text { y normal zació }\end{array}$ & $\begin{array}{c}\text { Contexto de la } \\
\text { información en } \\
\text { México I }\end{array}$ & $\begin{array}{c}\text { Contexto de la } \\
\text { información en } \\
\text { México II }\end{array}$ & \\
\hline $\begin{array}{c}\text { Análisis de } \\
\text { sistemas }\end{array}$ & $\begin{array}{l}\text { Automatzación } \\
\text { de unidades de } \\
\text { información }\end{array}$ & $\begin{array}{l}\text { Redes y } \\
\text { sistemas de } \\
\text { información }\end{array}$ & $\begin{array}{l}\text { Nuevas tecnologías } \\
\text { de } \\
\text { información }\end{array}$ & \\
\hline $\begin{array}{l}\text { Usuarios de } \\
\text { la información }\end{array}$ & $\begin{array}{l}\text { Fondos de } \\
\text { información y } \\
\text { referencia }\end{array}$ & $\begin{array}{l}\text { Metodología } \\
\text { de la } \\
\text { investigación }\end{array}$ & $\begin{array}{l}\text { Seminario } \\
\text { de investigación } \\
\text { bibliolecológica }\end{array}$ & \\
\hline & 'II)ADES DEP & VAS Y CULTU & & \\
\hline
\end{tabular}

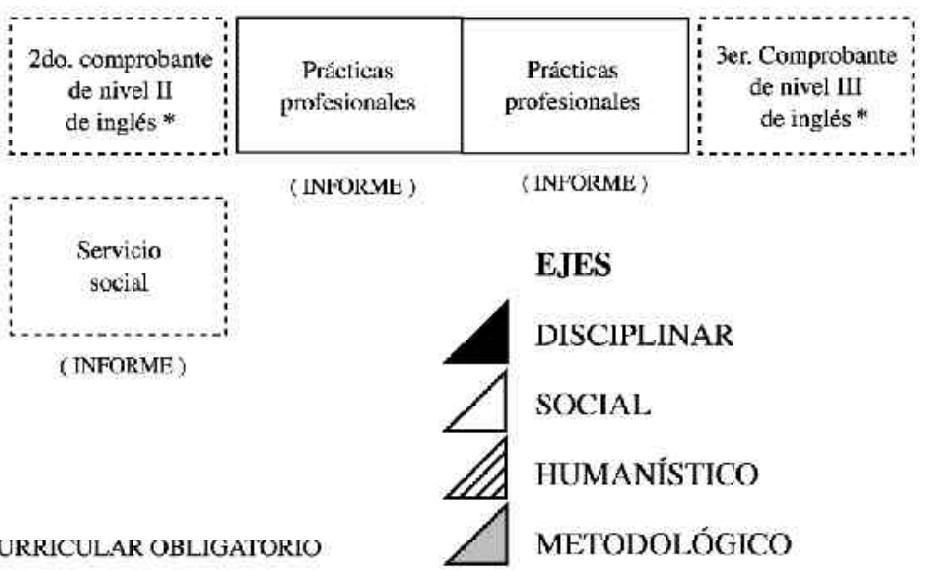




\section{CONSIDERACIONES GENERALES}

* El paradigma empíreo-analítico ha aportado importantes conocimientos al mundo moderno, la ciencia acumula el conocimiento a través de la investigación cuantitativa.

* Aunque se ha cuestionado el positivismo, éste proporciona una forma importante de acceder a un tipo de realidad.

* La investigación cuantitativa permite hacer pronósticos para la resolución de problemas y alcanzar la calidad en distintas actividades o procesos sociales.

* El paradigma es importante en la formación integral del licenciado en bibliotecología, así como para enriquecer y legitimar a nuestra disciplina como ciencia social.

\section{REFERENCIAS}

Bravo, Víctoreal. (1980). - MarxDurkheimyWeber:Teøńayrealidaden Méxica- México: Juan Pablos, pp. 13-22.

Budd, John M. (1995). - "An epistemological foundation for libraryand in formation scien ce." Thelibraryquartely65 (3): 295318.

Góméz,MireyayDulceMaríaPeralta(1995).- "Lasmátemáticas y las ciencias: Su vin culación con losnuevos pro gramas dees tudio."- Desded Sur. HumanismoyCienia(México) 1(3):37-55, diciembre.

Gorbea- Portal, Salvador(1995). - "Comen tario".- Investigaión Biblicteedógica (México) 9(18): 2-3, enero-junio.

ProyeetodkreestructuracoónamiaularparalaEsaudadeBibiotecoloǵaeln formacoóndelaUniversidadAutónomadeSanL uisPotoś:PlanInstitz donaldadesamollo1997-2007" "OfertaEducativayDiseñoCumiaular" I Rosa María Martínez Rider (Coord.), Salvador G orbea Portal (Asesor), Beatriz Rodríguez Sierra, Socorro Amada Mendoza Leos, Constanza A. Pérez Villega, María Magdalena Vázquez Sán chez, G riseldaPérez Gó mez,NormaLiliaAriceagaHernández, Agustín Gutiérrez Chiñas, Juan René GarcíaLagunas, Sergio Marcelino Núñez Pastrana- - San LuisPo tosí:Universidad Autónoma de San Luis Potosí, 1998.- 334 p.

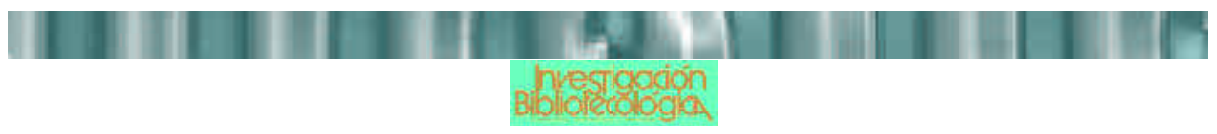

